\title{
Otto coupling to a transverse-electric-polarized mode on a metamaterial surface
}

\author{
Alastair P. Hibbins, ${ }^{*}$ Matthew J. Lockyear, and J. Roy Sambles \\ Electromagnetic Materials Group, Department of Physics and Astronomy, University of Exeter, Stocker Road, \\ Exeter EX4 4QL, United Kingdom
}

(Received 12 May 2011; revised manuscript received 27 July 2011; published 21 September 2011)

\begin{abstract}
Using the Otto geometry, prism coupling of microwave radiation to a metamaterial surface that supports a bound transverse-electric-polarized surface mode is demonstrated. The dispersion of this surface mode is characterized experimentally for incident radiation beyond the critical angle of the prism, and its resonant fields are explored using a numerical model.
\end{abstract}

DOI: 10.1103/PhysRevB.84.115130

PACS number(s): 41.20.Jb, 42.25.Bs

\section{INTRODUCTION}

The study of tightly bound electromagnetic (EM) waves guided at the interface between two dissimilar nonmagnetic media is well established. At the beginning of the 20th century, Cohn ${ }^{1}$ and Uller $^{2}$ appear to have been the first to investigate EM waves guided between a dielectric and a conductor with Zenneck ${ }^{3}$ and Sommerfeld ${ }^{4}$ subsequently realizing the application of their predecessors' proposals to the propagation of radio waves guided at the interface between two different media. These waves, which can be associated with the Brewster-angle phenomenon of zero reflection for transverse-magnetic- (TM-) polarized radiation, are interface modes with field amplitudes that decay exponentially with distance into the media either side. ${ }^{5}$ In the decades that followed, a large number of studies were undertaken by researchers involved in radio-wave and antenna-design technologies to understand the excitation and propagation characteristics of these modes across the conducting surfaces, such as the ground, the ocean, and metal objects (e.g. Refs 6-8). In parallel, work was being undertaken in the visible regime to comprehend the anomalous EM response of metallic diffraction gratings: dark bands in the reflected spectra observed by Wood $^{9,10}$ were eventually attributed to the resonant excitation of the same physical phenomena. The dispersion of the mode observed in the visible regime is now known to be characterized by the plasma frequency $\left(\omega_{P}\right)$ of the metal (typically in the ultraviolet) below which the real part of the metal's frequency-dependent permittivity is negative. At these frequencies, charge is induced at the metal surface in order to maintain a continuous normal component of the displacement field across the interface. The resonant frequency of the longitudinal oscillation of this induced charge density is known as the surface plasma frequency, $\omega_{S P}=\omega_{P} / \sqrt{2}$, for a planar air-metal interface. A net storage in magnetic energy at the surface results from the requirement of continuous tangential components of the electric field across the interface. The boundary condition at the surface of metals in the visible regime can therefore be described as predominantly inductive (albeit with a small resistive part). The surface mode is photonlike at low frequencies (i.e. it closely follows the light line, a grazing photon) but becomes strongly hybridized with the resonance of the surface plasma at higher frequencies and asymptotically approaches $\omega_{S P}$ (at which the relative permittivity of the metal, $\mathcal{E}_{\text {metal }}=-1$. The resulting mode is termed a surface plasmon polariton (SPP) and essentially represents the anticrossing phenomenon of the incident photon mode with the density oscillation resonance of the surface electrons. This surface wave (SW) is therefore a slow wave $\left(v_{S W}<c\right)$, nonradiative on a planar surface (i.e. $k_{S W}<$ $\omega / c)$, and for a planar nonstructured surface is transverse magnetic (TM) in polarization.

The extension of this SPP concept to the microwave and $\mathrm{THz}$ regimes would at first sight seem problematic since, at frequencies below the scattering rate of the electrons, metals behave as near-perfect conductors whose dielectric function has $\operatorname{Im}(\varepsilon) \gg|\operatorname{Re}(\varepsilon)|$, and thus the surface impedance has positive and almost equal real and imaginary parts, i.e. the small resistance of a metal surface is accompanied by an equally (small) value of inductance. This gives loosely bound surface waves which propagate almost without attenuation at speeds close to the speed of light (i.e. $k_{S W}=\omega / c$ ). Their fields extend for many wavelengths into free space and penetrate into the metal substrate by only a small fraction of the incident wavelength; therefore, these modes can be well described as simple surface currents. However, as early as 1944, workers became aware that the addition of a subwavelength corrugation to the metal (i.e. a metasurface) would produce an enhanced surface reactance and strongly bind the mode to the interface, even in this long-wavelength regime. For example, Barlow and Cullen ${ }^{6}$ and Cutler ${ }^{7}$ considered a corrugated metal slab in which each narrow groove can be regarded as a parallelplate transverse electromagnetic (TEM) transmission line. If the grooves are designed to be resonant, e.g. approximately one-quarter of a wavelength deep, then the short circuit (i.e. their closed end) at the bottom is transformed by the depth of the slot into an open circuit at the open end, such that the surface impedance becomes large in magnitude. However, if the depth of the slots is less than one-quarter wavelength, then the effective surface impedance is dominated by a large positive reactive term, and hence, its inductance is enhanced above that of a planar metal. Such surfaces are thereby able to support TM-polarized, bound surface waves that are cutoff at the resonant frequency. This characteristic frequency is analogous to the limiting surface plasma frequency $\left(\omega_{S P}\right)$ of metals in the visible regime.

By employing the principle of duality, ${ }^{11}$ it can be shown that Maxwell's equations also predict a bound transverseelectric- (TE-) polarized surface mode, provided that the two semi-infinite media constituting the interface possess opposite sign of relative permeability $\mu .{ }^{12}$ However, in contrast to the plethora of studies of TM-polarized surface waves 
discussed above, relatively few workers have presented studies of TE-polarized modes in the open literature. ${ }^{13-15}$ This lack of exploration can be easily understood by considering the requirement for a negative value of $\mu$, which is needed to satisfy the continuity equation for bound TE surface modes. For nonmagnetic materials, $\mu=1$, and for most magnetic materials $\mu>0$, except for ferromagnetic and antiferromagnetic media, which may possess a negative relative permeability over a narrow region in frequency close to their resonant frequencies. ${ }^{16}$ However, the recent surge of interest in either singly or doubly negative index metamaterials for use as superlenses, ${ }^{17}$ cloaking devices, ${ }^{18,19}$ or slow light devices ${ }^{20}$ has provided researchers an opportunity to explore the response of materials with exotic values of effective permeability $\mu_{\text {eff }}$. These effects are associated with the resonance of the metamaterial's subwavelength geometric structure (and may often be observed over a broader bandwidth and at higher frequencies than the inherent phenomenon associated with the resonance of ferromagnetic media). However, since permeability is a macroscopic quantity, in order to consider such metamaterials as possessing a meaningful $\mu_{\text {eff }}$, the metamaterial must comprise a large number of layers. ${ }^{21}$ For example, Ishikawa et al. ${ }^{22}$ have presented a numerical and analytical study which proposes that a deep subwavelength waveguide can be envisaged in which two of the waveguide walls are comprised of a material that behaves as if it has negative effective permeability. Furthermore, Gollub et al. ${ }^{12}$ recently showed that a thick, multilayer arrangement of splitring resonators could provide a negative $\mu_{\text {eff }}$ response and, hence, support TE-polarized surface waves around $10 \mathrm{GHz}$, termed there as a magnetic surface plasmon.

In this paper, we do not utilize a bulk or multilayered structure, and hence, the concept of a macroscopically defined EM property is not useful. However, in the same manner that a metasurface can present an inductive impedance and support TM-polarized surface waves, it is experimentally demonstrated here that TE-polarized modes can be supported on surfaces whose impedance is capacitive. We demonstrate prism coupling of incident TE-polarized microwave radiation to a strongly bound surface mode supported by a thin $\left(\ll \lambda_{0}\right)$ single-layer metasurface, in the form of a Sievenpiper mushroom-type structure. ${ }^{23}$ Recently, we showed that the same surface studied at a frequency lower than the fundamental resonant frequency (i.e. the region of inductive surface impedance) could support a TM-polarized surface mode at microwave wavelengths, similar to the spoof plasmon modes suggested by Pendry et al. $^{24}$ The TE-polarized surface mode is supported above the fundamental resonant frequency of the structure, where the surface impedance $Z_{s}$ is capacitive. ${ }^{25}$ As previously discussed, in a region of capacitive surface impedance, the metamaterial will present a boundary condition that is analogous to the way that a bulk medium dominated by a negative real $\mu$ behaves. The mode supported exhibits strong power flow at the surface of the sample and may be utilized in a similar manner to the previously reported TM-polarized surface wave.

\section{EXPERIMENTAL}

Formed from standard circuit board materials, the sample studied here consists of square, $18-\mu \mathrm{m}$-thick copper patches

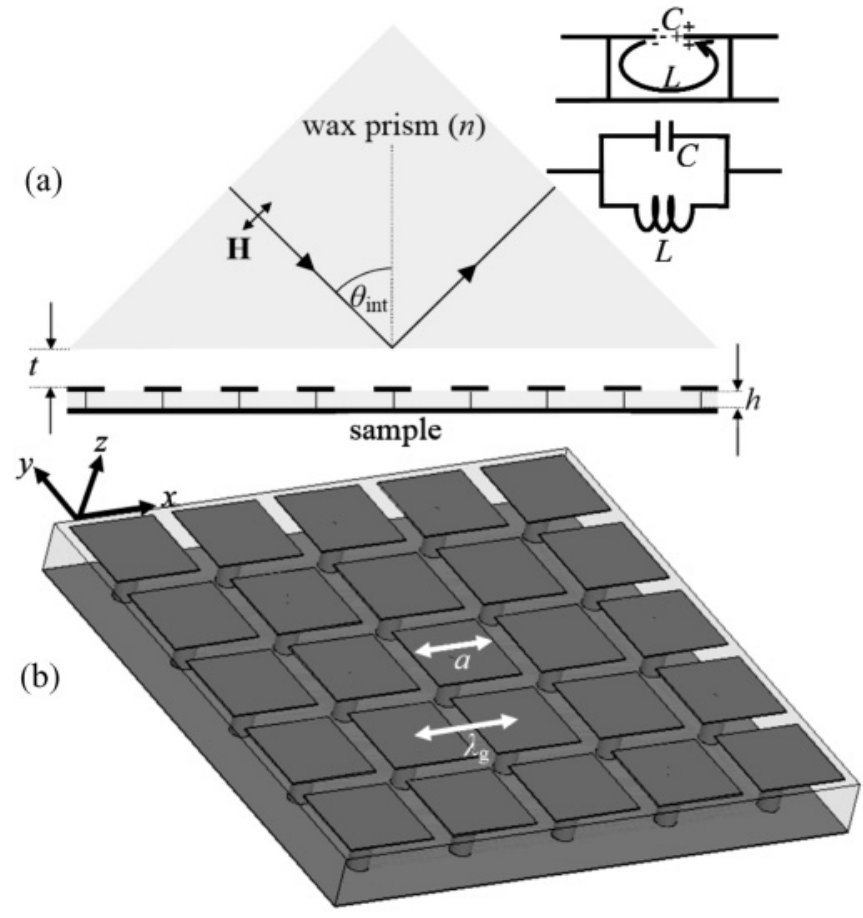

FIG. 1. Schematic representation of (a) the experimental geometry and (b) the sample under study. The inset shows an equivalent circuit model of the sample.

of side length $a=1.3 \mathrm{~mm}$ arranged in a square array of pitch $\lambda_{g}=1.6 \mathrm{~mm}$. The patches are separated from a copper ground plane by a low loss 0.79 -mm-thick dielectric [Nelco NY9220 $(\varepsilon=2.22+0.002 \mathrm{i})]$ with individual copper patches connected to the ground plane by a hollow copper via of radius $r_{v}=0.15 \mathrm{~mm}$, as shown in Fig. 1 . The features of the structure that constitute a unit cell are subwavelength $\left(\lambda_{g}=1.6 \mathrm{~mm}\right.$ compared to an incident wavelength range of $10<\lambda_{0}<$ $30 \mathrm{~mm}$ ) and thus may be represented by lumped circuit theory as a capacitor and inductor connected in series ${ }^{26}$ [Fig. 1(a) inset]. This simplified model can predict the EM response of the sample to first order with the capacitive component of the resonance arising from the capacitive coupling between nearest-neighbor patches and the inductive contribution from oscillating surface currents formed between the patches and ground plane through the vias.

Collimated plane wave microwave radiation is incident via a large wax prism $(n=1.48)$ at an angle beyond the critical angle $\left(\theta_{\text {int }}>\sin ^{-1} 1 / n\right)$, polarized with the incident magnetic field vector $(\mathbf{H})$ lying in the plane of incidence (transverse electric, TE-polarized) as shown by Fig. 1(a). Spacing the prism above the sample by a distance $t$ to provide a suitable tunnel barrier, the evanescent fields beyond the prism resonantly couple to the TE-polarized mode supported by the surface (Otto geometry ${ }^{27}$ ). Reflected radiation from the sample is collected using a 4-m radius of curvature spherical mirror and focused to a detector. The resonance is observed as a reflectivity minimum in the specular beam due to power being dissipated on resonance into the dielectric core and via Joule heating of the metal. Over a range of incident angles $\left(43^{\circ}<\right.$ $\theta_{\text {int }}<66^{\circ}$ ), the parameter $t$ is varied, allowing the optimum coupling condition to be found. For this optimum condition, 
the resonant frequency of the mode was evaluated allowing the in-plane wave vector $k_{x}$ to be extracted and the mode's dispersion to be experimentally constructed.

\section{RESULTS}

Figure 2 inset shows the typical reflectance obtained from the sample as a function of frequency for a range of tunnel gaps $(2 \mathrm{~mm}>t>5 \mathrm{~mm})$ with $t$ stepping in increments of $0.5 \mathrm{~mm}$. Here, TE-polarized radiation is incident via the wax prism at an internal angle $\theta_{\text {int }}=51.8^{\circ}$ with the resonant mode occurring at $51.3 \mathrm{GHz}$. It is important to note that this mode varies with frequency and coupling strength as a function of tunnel gap thickness $t$. Indeed, as $t$ is varied from $2 \mathrm{~mm}$ to $5 \mathrm{~mm}$, the resonance moves from an overcoupled state to an undercoupled state through an optimum coupling condition $\left(t_{\mathrm{opt}}=3.5 \mathrm{~mm}\right)$ when the radiative losses (i.e. outcoupling of the surface mode via the prism) are approximately equal to the nonradiative losses (i.e. Joule heating). The shift in resonant frequency of the mode for reducing $t$ is a result of increased penetration of the surface mode's exponentially decaying fields into the prism. ${ }^{28}$

In order to experimentally map the dispersion of the TEpolarized mode, the angle of incidence was fixed at 20 different values in the range $43^{\circ}>\theta_{\text {int }}>66^{\circ}$. For each value of $\theta_{\text {int }}, t$ was varied in $0.5-\mathrm{mm}$ increments. This allowed the resonant frequency of the TE-polarized mode to be extracted at the optimum coupling condition for each angle. The value of the corresponding in-plane wave vector $\left(k_{x}\right)$ was then calculated

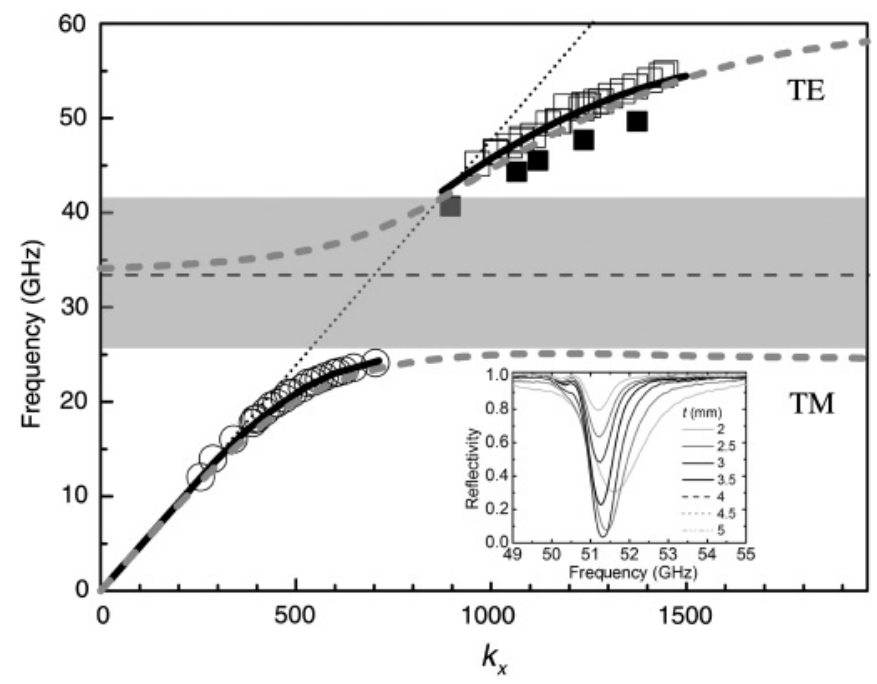

FIG. 2. The dispersion of the surface modes supported by the sample (shown in Fig. 1) derived from the frequency of the optimum coupling condition for a range of angles of incidence, $\theta$ (where $k_{x}=\omega / c \sin \theta$ is the component wave vector along the surface). The experimental data (open squares and circles for the TE- and TM-polarized modes, respectively) are compared to the predictions of the FEM modeling (solid line). The experimentally determined TE-polarized mode on the sample without vias is also shown (filled squares). The inset illustrates the reflectivity data recorded from the sample with $\theta_{\text {int }}=51.8^{\circ}$ for various values of $t$. The predicted dispersion of the surface eigenmodes of the sample without the influence of the coupling-in prism is also shown (dashed grey lines). The surface wave band gap is illustrated by the shaded region. and plotted against frequency [Fig. 2 (open squares)]. For completeness, the experimentally determined dispersion of the bound TM-polarized mode associated with the same surface is also shown (open circles) on this figure, and a discussion of its form may be found in Ref. 28. We firstly note the excellent agreement between the experimentally obtained dispersion of the TE-polarized mode (open squares) and that obtained via a finite element method (FEM) model (solid line). ${ }^{29}$ The modeled dispersion for the TE-polarized mode was obtained by considering a unit cell of the sample with plane-wave radiation incident through a dielectric medium $(\varepsilon=2.2+0.02 \mathrm{i})$ representing the experimental wax prism. A suitable tunnel barrier (air) was also modeled to accurately reproduce the experiment. The fundamental resonant frequency, $f_{\text {res }}$, for this sample (horizontal dashed line) is also shown, calculated from its geometrical parameters using an effective surface impedance model. ${ }^{25}$ This model considers a parallel connection of the impedance of the array of patches and the input impedance of a TEM line of length, $h$, and is valid in the limit $\lambda_{g}<\lambda_{0}$, and for normal incidence. Plotting $Z_{s}$ as a function of $k_{x}$ allows the resonant frequency of the structure to be obtained by observing the singularity in the expression at $f_{\text {res. }}$ Numerically solving this equation predicts $f_{\text {res }}=33.4 \mathrm{GHz}$ for the given sample parameters. ${ }^{28}$

The experimental TE-polarized response of the sample without the vias connecting each individual patch to the ground plane is also shown (filled squares). It is interesting to note that the response of the TE-polarized surface mode is only slightly perturbed in resonant frequency, but not form, by the removal of the vias. This reduction in frequency is associated with the removal of the fundamental resonant frequency of the structure at $33.4 \mathrm{GHz}$ and the associated surface wave band gap (which is symmetrically centered at this frequency, the lower frequency edge of which provides the asymptotic limit for the TM-polarized mode). Without the vias, the fundamental resonant frequency of the structure is determined to first approximation simply by the side length of the patch fulfilling a one-half resonant condition $(115.3 \mathrm{GHz}$ for this structure). However, although the TE-polarized surface mode is only slightly perturbed, the dispersion of the TM-polarized mode changes dramatically with the removal of the vias. This is because, without the band gap about $33.4 \mathrm{GHz}$, its dispersion would follow the light line, passing through the TE-polarized surface mode dispersion and continuing to its new limit at the Brillouin zone boundary where the group velocity must be zero. $^{30}$

From the dispersion obtained over the experimental range of frequency presented here, it is evident that the TE-polarized surface mode supported on metasurfaces both with and without vias exist in the nonradiative region (i.e. to the right of the light line). Also note that above the fundamental resonant frequency and band gap of the structure with vias, where $Z_{s}$ is capacitive (due to the fields being largely contained in the dielectric between nearest-neighbor patches), the dispersion curves away from the light line and becomes more tightly bound to the surface with increasing frequency (as indicated by the slope of the dispersion curve). 


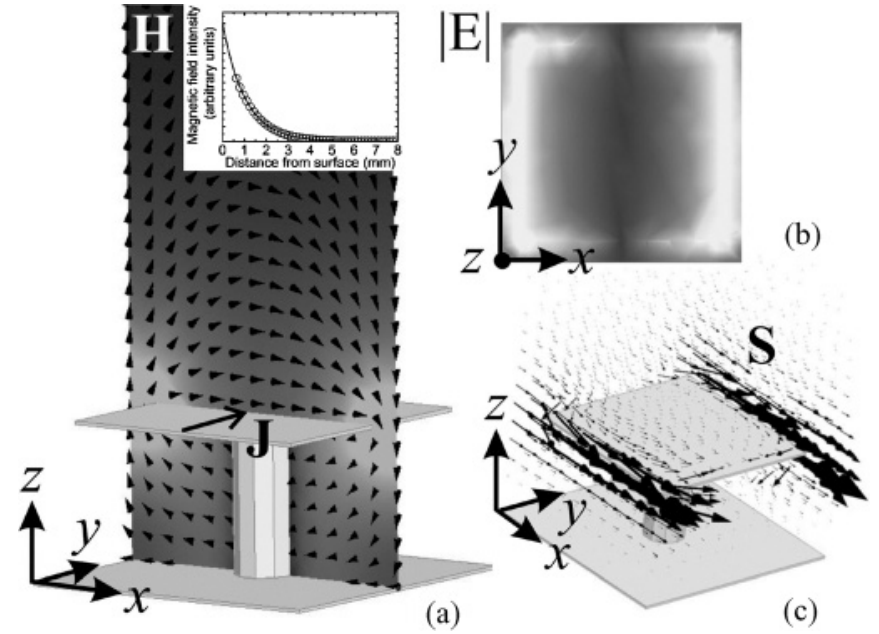

FIG. 3. (a) FEM model predictions of the vectorial magnetic field on resonance of the TE-polarized surface mode at $k_{x}=7 k_{g} / 18$, plotted over the $x z$ plane passing through the center of the patch at a temporal phase corresponding to maximum field enhancement. The plane of current, $\mathbf{J}$, that flows parallel to the $y$ direction along the surface of the patches together with the (b) time averaged electric-field enhancement and (c) Poynting vector are also shown. The inset of (a) illustrates the decay of the magnetic field intensity from the surface of the metamaterial (circles: FEM prediction; line: exponential fit with decay length $\sim 1 \mathrm{~mm}$ ).

\section{MODELING AND DISCUSSION}

To fully understand the nature of the surface modes supported by these structures, it is useful to calculate the mode dispersion using the eigenmode solver of the FEM model. This technique allows the dispersion of the surface modes to be determined over a range of in-plane momenta from $k_{x}$ $=0$, to $k_{x}=k_{g} / 2$ (the first Brillouin zone boundary). In addition, the requirement of a momentum-matching technique to facilitate the coupling of incident radiation to the surface mode is negated in this solver, thus removing any perturbation to the modes' dispersion due to the proximity of the wax prism to the sample. The grey dashed lines on Fig. 2 show the calculated dispersion of the TM- and TE-polarized surface modes supported by the sample with connecting vias. The light line (dotted line), $f_{\text {res }}$ (horizontal dashed line), and surface wave band gap (shaded region) are also shown. It is clear that the TM-polarized mode closely follows the light line for low values of $k_{x}$ before becoming asymptotic to the lower edge of the surface wave band gap, which is centered symmetrically around $f_{\text {res }}$ for this mushroom structure. For the TE-polarized surface mode, there is no such asymptotic limit. Instead, the dispersion of the TE-polarized mode curves away from the light line to approach the Brillouin zone boundary with zero group velocity. At this point, the surface wave is a standing wave, the frequency of intersection with the Brillouin zone boundary being largely determined by the periodicity of the surface, $\lambda_{g}$. Figure 3(a) shows the vectorial magnetic field on resonance of the TE-polarized surface mode at $k_{x}=$ $7 k_{g} / 18$, plotted over the $x z$ plane passing through the center of the patch. The TE-polarized nature of the mode is clearly evident with loops of magnetic field above and below the top surface of the metamaterial, confirming its capacitive surface impedance. This should be contrasted to the electric-field loops associated withTM-polarized surface waves on inductive surfaces ${ }^{28}$ including SPPs on real metals in the visible. ${ }^{31}$ For TM-polarized surface modes, it is essentially the electric dipoles (and higher-order modes) associated with the patches that comprise the metamaterial surface that couple to the incident radiation. Analogously, the TE-polarized surface mode that exists above the band gap is associated with the oscillating plane of current that flows parallel to the $y$ direction along the surface of the patches [Fig. 3(a)]. The magnetic field loops circulate around the line of current. This results in a strong power flow [Fig. 3(c)] at the surface along the slits between the patches in the $x$ direction, owing to the enhanced localized electric and magnetic [Fig. 3(a) inset] and electric [Fig. 3(b)] field intensity that is permitted in these regions at frequencies close to the geometrical resonance.

\section{CONCLUSIONS}

We have experimentally demonstrated that a thin metamaterial of the Sievenpiper mushroom type supports a strongly bound transverse-electric-polarized surface mode at microwave wavelengths. The dispersion of the mode is mapped experimentally and also modeled using a finite element method model. Joule heating of the metal and dielectric composite provides high absorption efficiency on resonance at a frequency which may be tuned via the geometry of the structure. Further, evaluation of the electromagnetic fields on resonance shows current flow along the patches and magnetic-field loops in the plane of incidence, resulting in strong power flow confined to the surface.

\section{ACKNOWLEDGMENTS}

The authors wish to thank the Engineering and Physical Sciences Research Council (EPSRC,UK) for continued financial support.

\footnotetext{
*a.p.hibbins@ex.ac.uk

${ }^{1}$ E. Cohn, Das Elektromagnetische Feld (Leipzig: S. Hirzel.), 2nd ed. (Berlin, 1927).

${ }^{2} \mathrm{~K}$. Uller, Beiträge zur Theorie der elektromagnetischen Strahlung (University Rostock, Rostock, 1903).

${ }^{3}$ J. Zenneck, A. Phys. 23, 846 (1907).

${ }^{4}$ A. Sommerfeld, A. Phys. 333, 665 (1909).

${ }^{5}$ J. A. Stratton, in Electromagnetic Theory (McGraw-Hill Book Company, New York, 1941), p. 509.
}

${ }^{6}$ H. M. Barlow, A. L. Cullen, G. G. Macfarlane, B. I. Stuart, J. G. Linhart, R. B. Dyott, and A. E. Karbowiak, Proc. IEE 100, 329 (1953).

${ }^{7}$ C. C. Cutler, in Antennas and Propagation Society International Symposium (AP-S. Digest Seattle, WA, USA, 1994).

${ }^{8}$ G. N. Watson, Proc. Roy. Soc. Series A 95, 83 (1918).

${ }^{9}$ R. W. Wood, Philos. Mag. 4, 396 (1902).

${ }^{10}$ R. W. Wood, Phys. Rev. 48, 928 (1935).

${ }^{11}$ C. A. Balanis, Proc. IEEE 80, 7 (1992). 
${ }^{12}$ J. N. Gollub, D. R. Smith, D. C. Vier, T. Perram, and J. J. Mock, Phys. Rev. B 71, 195402 (2005).

${ }^{13}$ K. Button and B. Lax, Antennas and Propagation, IRE Trans. Ant. Propag. 4, 531 (1956).

${ }^{14}$ L. Courtois, G. Declercq, and M. Peurichard, Magnetism and Magnetic Materials, AIP Conference Proceedings 5, 1541 (1971).

${ }^{15}$ M. R. F. Jensen, S. A. Feiven, T. J. Parker, and R. E. Camley, Phys. Rev. B 55, 2745 (1997).

${ }^{16}$ G. Suran, M. Naili, H. Niedoba, F. Machizaud, O. Acher, and D. Pain, J. Magn. Magn. Mat. 192, 443 (1999).

${ }^{17}$ J. B. Pendry, Phy. Rev. Lett. 85, 3966 (2000).

${ }^{18}$ J. B. Pendry, D. Schurig, and D. R. Smith, Science 312, 1780 (2006).

${ }^{19}$ U. Leonhardt, Science 312, 1777 (2006).

${ }^{20}$ K. L. Tsakmakidis, A. D. Boardman, and O. Hess, Nature 450, 397 (2007).

${ }^{21}$ D. R. Smith, S. Schultz, P. Markos, and C. M. Soukoulis, Phys. Rev. B 65, 195104 (2002).
${ }^{22}$ A. Ishikawa, S. Zhang, D. A. Genov, G. Bartal, and X. Zhang, Phys. Rev. Lett. 102, 043904 (2009).

${ }^{23}$ D. Sievenpiper, L. J. Zhang, R. F. J. Broas, N. G. Alexopolous, and E. Yablonovitch, IEEE Trans. Microwave Theory Tech. 47, 2059 (1999).

${ }^{24}$ J. B. Pendry, L. Martin-Moreno, and F. J. Garcia-Vidal, Science 305, 847 (2004).

${ }^{25}$ S. Tretyakov, Analytical Modeling in Applied Electromagnetics (Artech House Inc, London, 2003), chap. 6.

${ }^{26}$ N. Engheta and R. W. Ziolkowski, Metamaterials, Physics and Engineering Exploitations (Wiley, New York, 2006), chap. 11.

${ }^{27}$ A. Otto, Z. Phys. 216, 398 (1968).

${ }^{28}$ M. J. Lockyear, A. P. Hibbins, and J. R. Sambles, Phys. Rev. Lett. 102, 073901 (2009).

${ }^{29}$ HFSS, software version 13, Ansys Corporation. Canonsburg, PA, USA

${ }^{30}$ F. Yang, A. Aminian, and Y. Rahmat-Samii, Microw. Opt. Techn. Let. 47, 240 (2005).

${ }^{31} \mathrm{H}$. Raether, Surface Plasmons on Smooth and Rough Surfaces and on Gratings (Springer, Berlin, 1988), chap. 2, p. 4. 